\title{
Some Unique Statistical Properties of the Seismic Process in Mines
}

\author{
S. Lasocki AGH University of Science and Technology, Faculty of Geology, Geophysics and Environmental \\ Protection, Poland
}

\begin{abstract}
From a statistical point of view, a series of seismic events is seen as the n-element sample from a stochastic process. If this process is stationary and memoryless then the complete information on the parameters of seismic events is preserved in the joint probability distribution functions of the parameters. In common practice the joint probability description is replaced with marginal distributions of a particular parameter. Such an approach through the marginal distributions splits the seismic process into a number of respective processes of the parameters of seismic events. The parameters can either be measurable, for example, the occurrence time, epicenter coordinates, focal depth, magnitude etc., or derived, for example, inter-event time, inter-event distance, maximum magnitude etc. Each of these parameter processes can be studied separately.

This paper reviews recent investigations into the statistical properties of the parameter series of seismic events from mines. Because the seismic process in mines is controlled by complex and changeable anthropogenic factors it is not surprising that the differences in statistical properties between mininginduced seismicity (MIS) and natural seismicity are substantial. Unlike tectonic seismicity, which is permanent in both space and time, MIS is transient and forms time-space zones or clusters that correlate in space and time with mining works. The event occurrence process in mines is not Poissonian. It is timedependent and, at best, can be regarded as quasi-stationary. Information on non-stationarity is stored mainly in smaller and more frequent events. The event magnitude processes in MIS is also time-dependent. Consequently, the Gutenberg b-value, the return period and other hazard parameters vary in time. Furthermore, for the majority of mining event parameterisations, their stochastic features cannot be ignored. The interval estimation of autocorrelation function used to study short-term interrelations, and the Hurst rescaled-range analysis applied to investigate long-term clustering, show that the occurrence process, the magnitude process and likely the event location process, have memory of both the long-and the short-type. Inter-event times, inter-event distances and magnitudes are internally interrelated. One of the possible ways for these interactions among mining-induced seismic events is the static Coulomb stress transfer. The subsequent events tend to locate within the areas of increased Coulomb stress due to previous events. Finally, the magnitude distribution of mining seismic events is complex and often multi-modal. Populations of magnitudes consist of at least two components. The magnitude distribution cannot be accurately approximated with the Gutenberg-Richter model.
\end{abstract}

The unique features of the MIS process presented: time-dependence, memory, inter-relations, multi-modality etc., show that the process is complex. This complexity complicates the practice of the statistical analysis of mining seismic data. On the other hand, however, the non-stationarity and inter-relations mean that the mining seismic event generation process is intrinsically predictable.

\section{Introduction}

Within the statistical approach to seismicity the time-ordered series of seismic events is a sample from an event generating stochastic process. The seismic events are described by a number of observables. Occurrence time, epicentre coordinates, focal depth, magnitude, seismic energy are the most common parameterisations of both natural as well as induced seismicity. These observables are later used to construct other parameters of the process, e.g. inter-event time, inter-event distance, maximum magnitude, activity rate etc. Some of such derived parameters, like the maximum magnitude and activity rate from the above examples, are connected with an observation period of selected constant length rather than with particular events. However, others, like the inter-event time or distance, can be linked to the consecutive events. Statistically all these parameters are random variables. Thus, the seismic process is represented by a time 
series of a multi-dimensional random variable, whose dimension equals the number of all the measureable and derived parameterisations of the seismic events.

It is commonly assumed that the basic parameters of seismic events: occurrence time, location coordinates and magnitude are mutually independent (Lomnitz, 1974). Under this assumption the multivariate representation of a seismic process is replaced with a set of separate processes of particular event parameters. In this paper, we summarise selected investigations into the statistical properties of the processes of the parameters of mining seismic events. This review shows that the dependence of mining seismicity on complex and changeable anthropogenic factors causes the seismic process in mines to have unique features, substantially different from the properties of the tectonic earthquake process. The processes of MIS parameters are in general time-dependent, and often have memory. Furthermore, the probability distribution of the magnitude of mining seismic events is distinctly multi-modal, which suggests that MIS is a mixture of the outcomes from different fracturing mechanisms. The consequences of this complexity of the MIS process for process predictability are discussed.

\section{Non-stationarity of MIS occurrence process}

Probably the most obvious difference between the generation processes of tectonic earthquakes and mining seismic events is their extension in time. Compared to the lifetime of humanity, the earthquake process duration is infinite. On the contrary, mining induced seismicity is controlled mostly by mining works and, being such, is transient rather than permanent. Mining seismicity forms well-defined time-space clusters or zones that correlate in both time and space with time-varying mining works (Orlecka-Sikora and Lasocki, 2002). Figure 1 shows the spatial distribution of the epicentres of mining events recorded in copper mines of the Legnica-Głogów Copper District (LGCD) in Poland in two consecutive six year periods: 1995-2000 and 2001-2006. The clustering of events and the changes of the locations of the active zones in time are distinctly visible.

The fact that the earthquake process is permanent and is controlled by factors that are constant in time led to an acceptation of the assumption that the main earthquake occurrence is Poissonian, i.e. stationary and memoryless (Gardner and Knopoff, 1974). Although inter-relations among earthquakes have been recently studied (Gomberg et al., 2005; Steacy et al., 2005), this assumption is still approved in many applications of statistics to seismology. For a Poissonian process, the event rate is governed by the Poisson distribution, which determines the probability of the occurrence of $n$ events in the time period of $D$ time-units:

$$
\operatorname{Pr}\{N=n ; D\}=\frac{(\lambda D)^{n}}{n !} \exp [-\lambda D]
$$

and the inter-event time, $\tau$, is exponentially distributed:

$$
F_{\tau}(\tau)=1-\exp (-\lambda \tau)
$$

where $N$ is the number of events to occur within a period of $D$ time units, $F_{\tau}(\tau)$ is the cumulative distribution function of the inter-event time, and $\lambda$ is the mean event rate; $\lambda$ is regarded as the process parameter. 

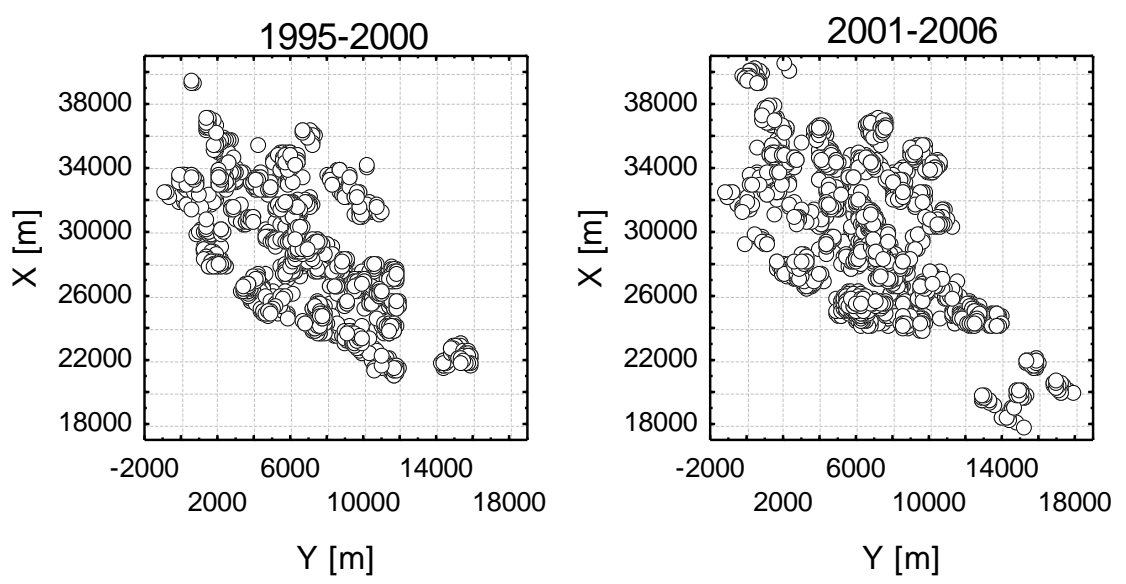

Figure 1 The distribution of the epicentres of mining events recorded in LGCD in two consecutive six year periods

The validity of the assumption of the Poissonian character of the MIS occurrence process was studied in 1992 (Lasocki, 1992). Two null hypotheses were tested: one that the numbers of events from constant length time periods follow the Poisson distribution and another that the inter-event time distribution is exponential. Two goodness-of-fit tests were used: the chi-square and the Kolmogorov-Smirnow test. The test data were four seismic event series recorded close to longwall stopes in underground coal mines in Poland. The series were numerous: 741 events recorded in 351 days, 2066 events recorded in 295 days, 700 events recorded in 365 days and 107 events recorded in 212 days, and comprised statistically all events from magnitude $\mathrm{M}_{\mathrm{L}}=0.7$. In all cases, the low significance of the null hypotheses strongly suggested that the event occurrences were not Poissonian. The same tests performed on sub-series from 50 day time periods led, however, to a considerable increase of the null hypotheses significance and the Poissonian character of the seismicity generation process could not be excluded. Moreover, the probability that the event occurrence process was Poissonian increased above reasonable rejection limits also for those sub-series consisting of only larger events. Similar studies of seismic event occurrences in South African gold mines were presented by De Toit at the Induced Seismicity workshop during the last IASPEI assembly in Perugia in 2007 (Du Toit and Mendecki, 2007). In some instances, the non-Poissonian structure of seismic event series was suggested.

Three conclusions can be drawn from the presented results. Firstly, the non-Poissonian character of seismicity is due to non-stationarity of the occurrence process rather than caused by the inter-relations of the event occurrences. Indeed, if the latter were true, then short samples should also be non-Poissonian. The non-stationarity means that the mean event rate, $\lambda$, changes in time and this phenomenon is, in fact, well-documented. Figure 2 shows an example of the distinct and systematic time-variability of the rate of mining seismic events. The seismic data was recorded in a region of mining stope in an underground copper mine in Poland. The activity rate values were evaluated from consecutive 30 event samples and linked to the middle of the sampling periods, respectively. The thick horizontal line indicates the mean activity rate value, equal to the 1.8 event/day that was calculated from the number of all events recorded in the period of observations. In the first period of observation, the seismic activity of the studied mining region was low. In the second part the activity was much bigger, occasionally greater than twice the average.

Secondly, the trend towards stationarity when the time period, over which event series are collected, is reduced suggests that the MIS occurrence process is quasi-stationary. Variation in the time of the event rate seems to be slow enough to enable the collecting of samples that are acceptably stationary and satisfactorily numerous to be used in statistical estimation tasks.

The third conclusion that arises from the fact that removal of smaller events simplifies the structure of an event series so that it becomes Poissonian, points out to the importance of small events in providing information about fracturing processes in mines. 


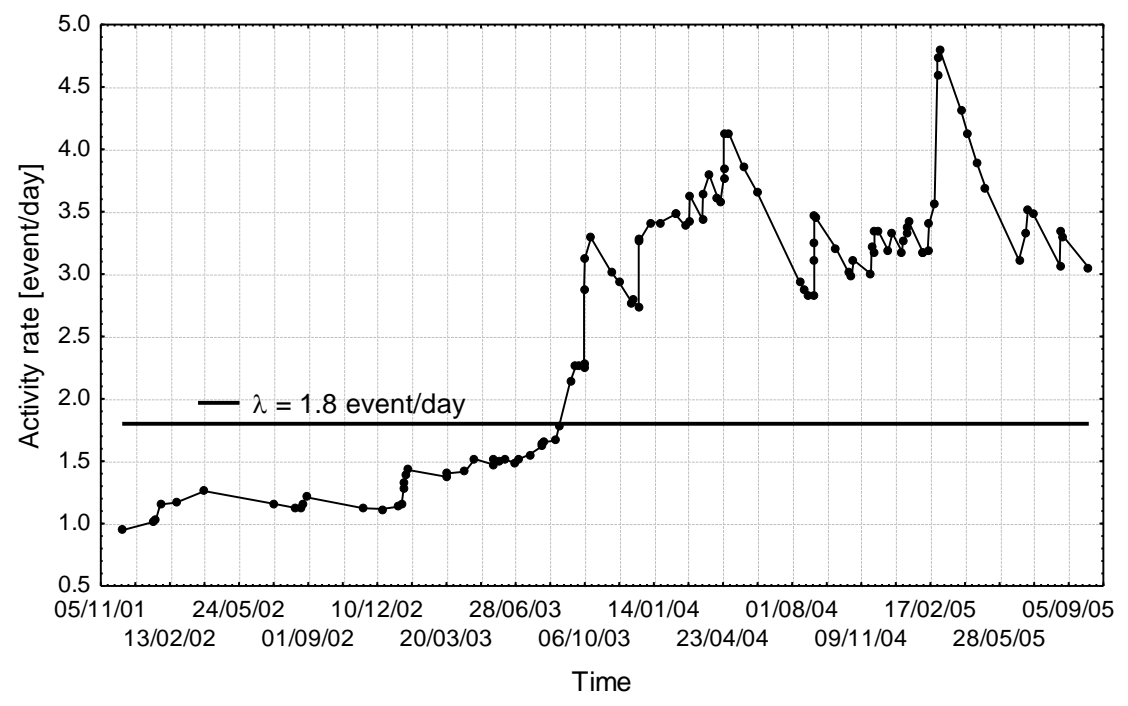

Figure 2 Time-variations of seismic activity of a mining stope region

\section{Non-stationarity of MIS magnitude process}

The Gutenberg-Richter relation, which describes a statistically linear decrease in the logarithm of the number of events larger than the given magnitude $m$ with increasing value of $m$, is probably the most used and over-used model for magnitude of seismic events in mines. Apart from the fact that a detailed insight into the probabilistic structure of magnitude populations is an evidence that this model is oversimplified for certain applications, which is addressed in a subsequent section, the model is good enough to monitor the time changes of the magnitude process. The mentioned form of the Gutenberg-Richter relation is:

$$
\log N(m)=a-b m
$$

where $a$ depends on the activity rate and the Gutenberg $b$-value determines a proportion of a larger events in the whole population. Changes of $b$ in time mean changes of the magnitude distribution, hence the nonstationarity of the magnitude process.

Observations of the significant time variations of the Gutenberg-Richter $b$-value in mining-induced seismicity go back more than thirty years. The phenomenon is well-documented world-wide and we mention it here only to ensure the continuity of the exposition. Some discussions of this phenomenon and relevant citations can be found in Gibowicz and Lasocki (2001) and the references therein. An example of changes of $b$ in time is presented in Figure 3. The seismic data was recorded in a region of exploitation stope in an underground copper mine in Poland. The event series was split into consecutive samples of 50 elements each. These samples were then used to calculate $b$-values with the maximum likelihood estimation procedure. The thick horizontal line indicates a $b$-value equal to 0.53 obtained from all magnitudes of the considered series of seismic events. Between November 2004 and June 2005, $b$ was increasing, which meant a decreasing probability of larger magnitudes occurrence. Later, between July and September 2005 the trend was reversed. During the last period of about one month, $b$ maintained a more or less constant, relatively high value. In general $b$-values were low, much less than 1.0 , which is sometimes considered a universal constant value of $b$ for earthquake processes (Kagan, 1999). Large deviations of $b$-values from 1.0 in both directions are typical for mining induced seismicity.

The obvious interpretation of the $b$-value in terms of the potential of the fracturing process to generate stronger seismic events and the time-variability of $b$ in MIS caused some popularity in the use of temporary $b$-estimates to monitor the seismic hazard in mines. It is worth commenting here that the sole $b$ value is not representative for the seismic hazard because it informs only of the distribution of the sizes of oncoming events with no regard to the chance that the events will occur at all. Occasionally an increase of $b$ can be compensated with the respective increase of the event rate and the probability of a larger event occurring can remain unchanged or even can increase. Therefore, to monitor the actual variations of the seismic hazard, the analysis of the $b$-value should be integrated with the analysis of the event rate (Lasocki, 1993). 
Figure 4 shows time variations of the seismic hazard for the same data as that used to exemplify the changes in the time of the $b$-value in Figure 3. The hazard is expressed by the return period of magnitude $m_{p}$, defined as:

$$
T\left(m_{p}\right)=\left\{\lambda\left[1-F_{m}\left(m_{p}\right)\right]\right\}^{-1}
$$

where $\lambda$ is the event rate and $F_{m}(\bullet)$ is the cumulative distribution function of the magnitude. Smaller values of $T$ indicate bigger hazards. In the presented example, the cumulative distribution of magnitude resulted from the Gutenberg-Richter relation (Equation 3), and the $\lambda$ and $b$ parameter values and consequently $T$ were calculated from the mentioned 50 event samples. The graph shows the time changes of the return period of $m_{p}=3.5$ events. In general, Figures 3 and 4 are similar but the differences caused by the changes of the activity rate are also distinct.

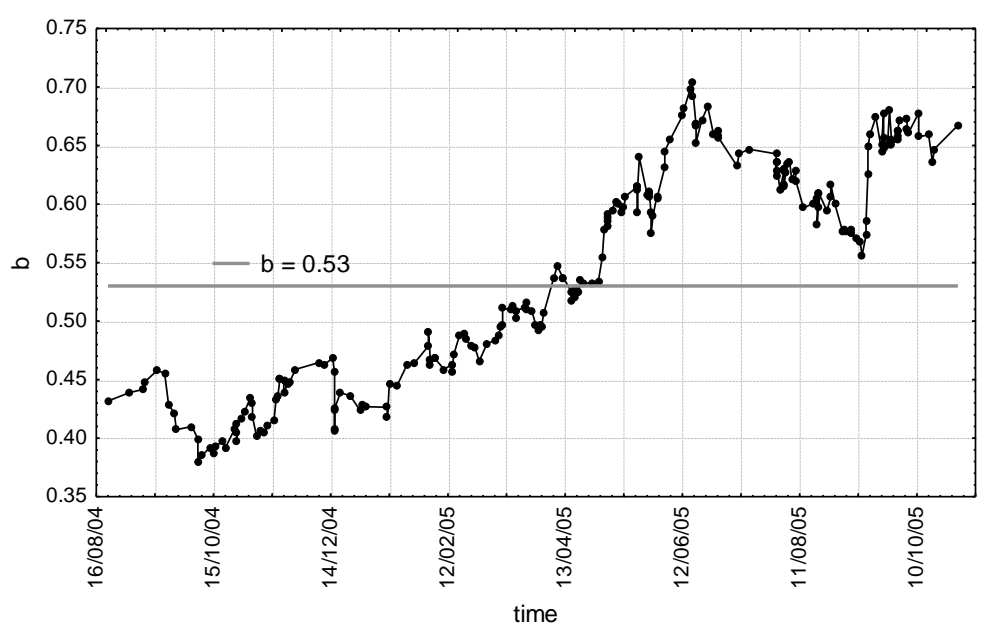

Figure 3 Time-variations of the Gutenberg $b$-value of seismicity of a mining stope region

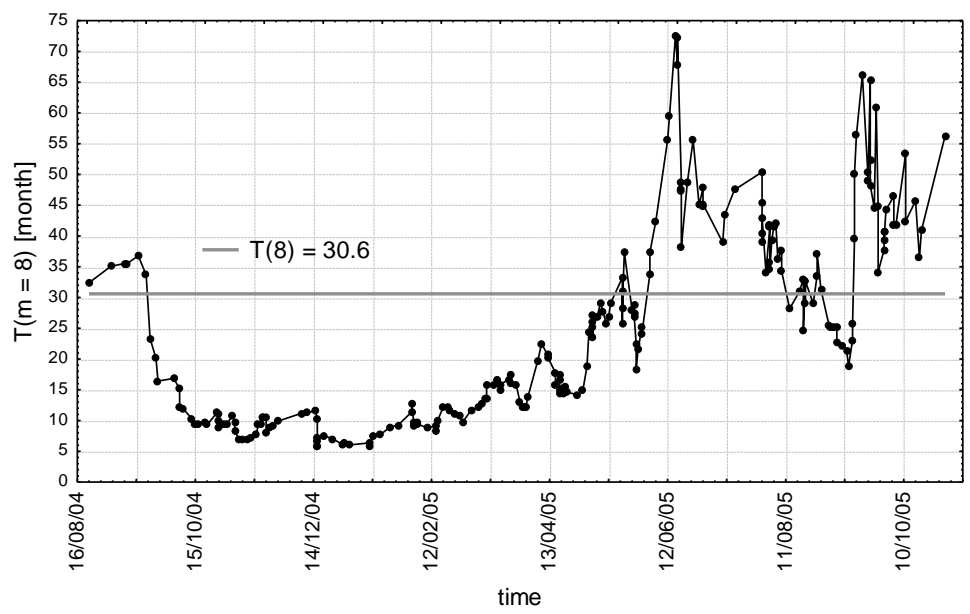

Figure 4 Time-variations of the seismic hazard, obtained for the same seismic data as used to calculate $b$-values presented in Figure 3. The hazard is quantified with the return period of events of magnitude 3.5

\section{Memory in MIS parameter processes}

A stationary stochastic process can have long- and/or short- memory or eventually none, if it is fully random. Węglarczyk and Lasocki (2008) searched for the memory in MIS parameter processes with the use of two methods: the Hurst rescaled range analysis (Hurst, 1951) to test for long memory, and the auto-correlation analysis to study the short memory property. 
Let $\left(X_{1}, \ldots, X_{n}\right)$ be a sample from a stochastic process $\mathcal{X}$. The rescaled range (R/S analysis) begins with splitting the sample into $d$ non-overlapping, consecutive intervals, each of the same length $s=n / d$. For every interval $k, k=1, . ., d$, the local range $R_{k}$ and its standard deviation $S_{k}$ are calculated as:

$$
\begin{gathered}
R_{k}=\max _{i=1, . ., s}\left\{Y_{i}\right\}-\min _{i=1, . ., s}\left\{Y_{i}\right\} \\
S_{k}=\sqrt{\frac{1}{s-1} \sum_{i=1}^{s}\left(Y_{i}-\bar{Y}\right)^{2}}
\end{gathered}
$$

where $Y_{i}=\sum_{j=1}^{i}\left(X_{j}-\bar{X}\right), i=1, . ., s ; \bar{X}=\frac{1}{s} \sum_{i=1}^{s} X_{i}$ and $\bar{Y}=\frac{1}{s} \sum_{i=1}^{s} Y_{i}$

The rescaled range is defined as:

$$
(R / S)_{s}=\frac{1}{d} \sum_{k=1}^{d} R_{k} / S_{k}
$$

The rescaled range is connected with the interval length $s$. Hurst proved that in the asymptotic case the logarithm of the rescaled range scales linearly with the logarithm of $s$ :

$$
\log \mathrm{E}\left[(R / S)_{s}\right]=H \log s+\text { const }
$$

where $\mathrm{E}[\bullet]$ stands for the expected value and $H$ is the Hurst coefficient. The straight line slope coefficient $H$ is greater than 0.5 for a persistent stochastic process, equal to 0.5 for a random walk (memoryless) process, and less than 0.5 for an antipersistent process. In practical applications, however, when the asymptotic conditions are not satisfied, the $H$ value estimator for a memoryless process is biased to an unknown quantity. Furthermore, $(R / S)_{s}$ evaluated from one sample, deviates randomly from $\mathrm{E}\left[(R / S)_{s}\right]$. Thus it is not known, which value of the $H$ estimate signifies the non-randomness of the process, and which could still result from a memoryless process, the estimator bias and the random error of estimation.

To remedy the problem, the authors of the cited work reproduced the empirical distribution of the Hurst coefficient estimator for a memoryless twin of the studied process $\mathscr{X}$ using bootstrap replicas of the sample $\left(X_{1}, \ldots, X_{n}\right)$. Bootstrapping, that is $n$ times random sampling with replacement from $\left(X_{1}, \ldots, X_{n}\right)$ cuts casual links among the process outcomes hence the estimation of $H$ from a bootstrap sample retains all the properties of the estimation of $H$ from the original sample but the $H$ estimate is relevant for the memoryless process.

The auto-correlation analysis can indicate the short memory of a stochastic process. The auto-correlation function is:

$$
A C C(l)=r_{l}=\frac{\sum_{i=1}^{m}\left(x_{i}-\bar{x}\right)\left(x_{i+l}-\bar{x}\right)}{\sum_{i=1}^{m}\left(x_{i}-\bar{x}\right)^{2}}
$$

where $l$ is the lag, $m=n-l$, and the overall mean is given by $X=\sum_{i=1}^{n} \frac{x_{i}}{n}$. When the studied process $\mathscr{X}$ has memory then $A C C(l)$ significantly deviate from zero for a number of the first lags.

The auto-correlation analysis of one sample from the process faces similar difficulties as the R/S analysis in determining the significance of inference, for which an interval estimation of $A C C(l)$ is needed. This time, however, to solve the problem the standard resampling procedures of $A C C(l)$ values are of no use because $A C C(l)$ values are not independent. Węglarczyk and Lasocki (2008) evaluated the interval estimates of the auto-correlation function from $A C C(l)$ replicas obtained by means of a special resampling procedure, suitable for dependent samples, called the blocks of blocks bootstrap. 
Fourteen seismic event series from different mining stopes from a copper mine of LGCD in Poland were analysed. The series were selected from visually stationary activity periods. Two parameter processes were investigated: the inter-event time, i.e. the event occurrence process and the magnitude process. The results of the analysis are provided in Table 1 . This contains the Hurst coefficient estimates ( $H$ column) and the autocorrelation marks ( $A C C$ column). The $H$ values in bold are those which were greater than the upper limit of the 99 per cent confidence interval of $H$ for the memoryless process. The $A C C$ marks were issued upon visual inspection of the interval estimates of $A C C(l)$. The double plus sign $(++)$ denotes very strong, the single plus (+) strong, the plus-minus (+-) questionable, and the minus (-) no evidence of deviation of the auto-correlation function from the zero line. With only two exceptions for the inter-event time, and three for magnitude the R/S study convincingly evidenced long memory in the studied processes. The short memory of these processes is not so evident, but for most of the considered cases it cannot be excluded.

Kijko (1997) studied inter-relations among seismic event occurrences in one of the deep gold mines in South Africa. For this purpose he was comparing the empirical cumulative distribution function of inter-event time $F_{\mathrm{OBS}}(\tau)$ with the cumulative distribution function of the exponential distribution of the Poisson process, $F(\tau)=1-\exp (-\lambda \tau)$. The positive values of the difference $D(\tau)=F_{\mathrm{OBS}}(\tau)-(1-\exp (-\lambda \tau))$ for certain inter-event times $\tau$ signify a trigger effect for these $\tau$-s: the events tend to recur sooner than in a random Poisson process. Conversely, if $D(\tau)<0$ for certain $\tau$-s then a reverse trigger effect for these $\tau$-s occurs, in which one has to wait longer for the next event than he would wait if the occurrence process were Poissonian. It was not surprising that the trigger effect appeared for very short inter-event times, up to about one hour, when the complete series of 19,889 events collected in one year was considered. This effect was connected with aftershocks whose non-random occurrence is a well known fact. However, the trigger effect was also found in the series comprising only 94 larger events, with apparent stress exceeding $100 \mathrm{MPa}$. Figure 4, taken from Kijko (1997), presents the $D(\tau)$ values versus the inter-event time, $\tau$ for this larger event series. The trigger effect extends over the inter-event times for up to 9 days and evidences inter-relations among the event occurrences.

Table 1 The results of $\mathbf{R} / \mathrm{S}$ analysis and auto-correlation analysis of mining series from the exploitation panels of a copper mine in Poland

\begin{tabular}{cccccc}
\hline \multirow{2}{*}{$\begin{array}{c}\text { Series } \\
\text { No. }\end{array}$} & $\begin{array}{c}\text { Number of } \\
\text { Elements }\end{array}$ & \multicolumn{2}{c}{ Inter-event Time } & \multicolumn{2}{c}{ Magnitude } \\
\cline { 3 - 6 } & & $H$ & $A C C$ & $H$ & $A C C$ \\
\hline 1 & 1200 & $\mathbf{0 . 8 5 2}$ & ++ & $\mathbf{0 . 7 8 2}$ & ++ \\
2 & 200 & 0.751 & + & 0.695 & - \\
3 & 1800 & $\mathbf{0 . 7 9 0}$ & +- & $\mathbf{0 . 6 5 4}$ & ++ \\
4 & 1500 & $\mathbf{0 . 7 8 3}$ & ++ & $\mathbf{0 . 6 5 2}$ & - \\
5 & 500 & $\mathbf{0 . 8 3 8}$ & +- & $\mathbf{0 . 7 5 7}$ & ++ \\
6 & 400 & $\mathbf{0 . 8 8 3}$ & +- & 0.603 & - \\
7 & 2600 & $\mathbf{0 . 8 4 4}$ & +- & $\mathbf{0 . 7 5 1}$ & +- \\
8 & 2200 & $\mathbf{0 . 8 0 1}$ & +- & $\mathbf{0 . 7 0 5}$ & +- \\
9 & 600 & $\mathbf{0 . 8 3 8}$ & +- & $\mathbf{0 . 7 2 7}$ & - \\
10 & 1100 & $\mathbf{0 . 8 2 8}$ & +- & $\mathbf{0 . 7 5 4}$ & +- \\
11 & 800 & $\mathbf{0 . 7 0 5}$ & +- & $\mathbf{0 . 6 5 4}$ & - \\
12 & 2600 & $\mathbf{0 . 7 4 4}$ & +- & $\mathbf{0 . 7 0 9}$ & ++ \\
13 & 360 & 0.673 & - & 0.607 & - \\
14 & 700 & $\mathbf{0 . 7 3 3}$ & +- & $\mathbf{0 . 7 0 2}$ & - \\
\hline
\end{tabular}




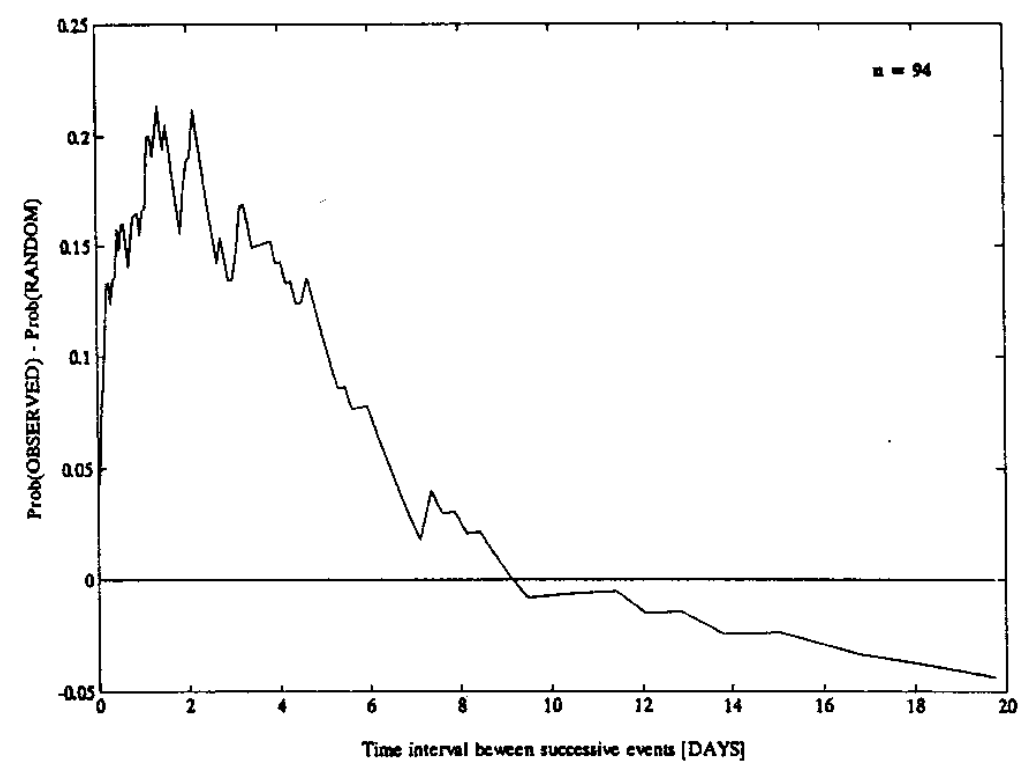

Figure 5 Difference between the empirical cumulative distribution function of inter-event time and the cumulative distribution function for the Poisson model of event occurrence as a function of the inter-event time (from Kijko (1997), Figure 8, page 252. Reprinted from: Gibowicz, S.J. and Lasocki, S. (editors), Rockbursts and Seismicity in Mines - Proceedings of the $4^{\text {th }}$ International Symposium, Krakow, Poland, 11-14 August 1997. A.A. Balkema, Netherlands, 1997)

\section{Stress transfer in MIS}

The previous sections show that the seismic occurrence and magnitude processes in mines are neither stationary nor memoryless. The likely origin of non-stationarity is clear: it is due to the link of the event generation to time-changeable mining works. In contrast, interaction among mining seismic events can arise for different reasons, which cannot be readily and unequivocally identified.

One of the considered mechanisms of earthquake interaction is a static stress transfer. Chinnery (1963) has shown that shear stress rises on an area larger than just the fault. The subsequent studies have presented the influence of the static stress changes on the space-time patterns of seismicity (Smith and Van de Lindt, 1969; Stein and Lisowski, 1983; Reasenberg and Simpson, 1992).

The static stress change due to a seismic event is expressed in terms of the Coulomb Failure Function (CFF). In short, the change of CFF on the fault plane of a subsequent, the so-called observing earthquake caused by the preceding earthquake is:

$$
\triangle C F F=\tau+\mu^{\prime} \sigma
$$

where $\tau$ is the shear stress in the slip direction on the fault plane of the observing earthquake and $\sigma$ is the normal stress on this plane, implied by the preceding event, and $\mu^{\prime}$ is the effective coefficient of friction $(0-0.4) . \tau$ and $\sigma$ are calculated from dislocation models of the preceding seismic source in an elastic and homogeneous half-space (King and Cocco, 2001). The observing fault is the one, whose occurrence could be triggered by the preceding event. The change in shear stress is positive for increasing shear stress in the direction of relative slip on the observing fault, the normal stress is positive for increasing tensional normal stress. Positive $\triangle C F F$ moves the fault toward failure, negative $\triangle C F F$ moves it away from failure. A triggering effect is expected when $\triangle C F F>0.01 \mathrm{MPa}$, which is only a small fraction of the corresponding stress drop.

Seismicity accompanying mining exploitation has been qualified as induced seismicity (McGarr and Simpson, 1997). Mining induced events occur in response to stress changes that are comparable to typical 
earthquake stress drops. Orlecka-Sikora et al. (2008) have, however, demonstrated that the stress transfer can also influence the mining seismic process. The authors studied the series of 768 larger events $\left(\mathrm{M}_{\mathrm{L}} \geq 2.0\right)$ from the Legnica-Głogów Copper District (LGCD) in Poland, from 1993-2006. They calculated the Coulomb stress changes that had taken place after the occurrence of every consecutive event, inverting each time the derived stress field according to the faulting type of the next event in the series. The events distinctly tended to locate within the areas of increased Coulomb stress due to previous events though a statistical confidence of these results has not been provided yet. Figure 5 shows snapshots of $C F F$ transfer in the studied area at a depth of $0.9 \mathrm{~km}$ for the two events. White indicates no significant $C F F$ changes before the events, grey regions denote negative changes in Coulomb stress and a decreased likelihood of fault rupture, and the black regions represent positive $\triangle C F F$ and an increased likelihood of rupture. The stars that mark the epicenters of the subsequent events are located in the relatively small black $\triangle C F F$ areas.
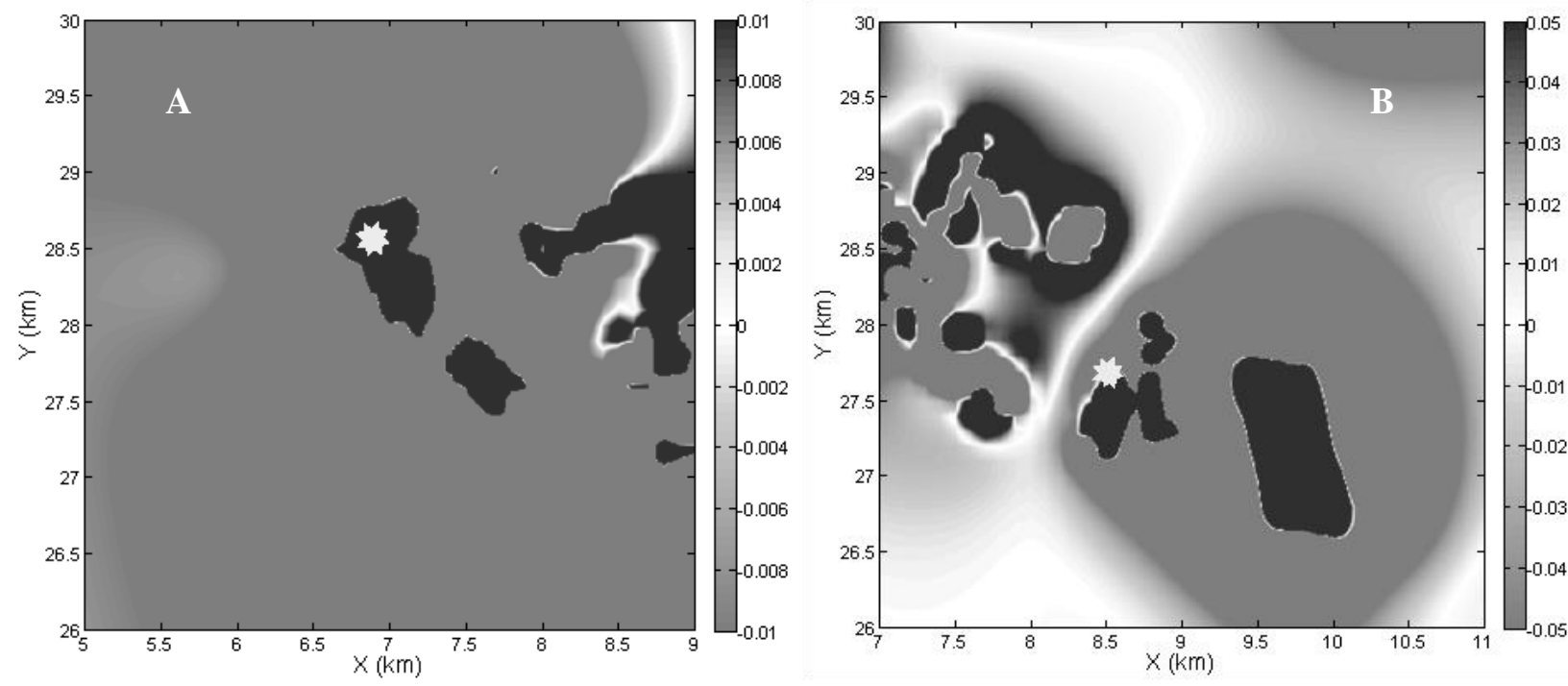

Figure 6 Coseismic Coulomb stress changes associated with the seismicity in the Rudna copper mine in LGOM. A) $\triangle C F F$ due to events preceding the event $M_{L}=3.6$ from 23.11.1995. The location of this event is marked with a star. B) The same as $A$ for the event $M_{L}=3.3$ from 22.09.1998. $\triangle C F F$ was calculated for faults at a depth of $0.9 \mathrm{~km}$ and for the faulting types of these two considered events, respectively

\section{Multi-modality of MIS magnitude populations}

Reports on breaks in the Gutenberg-Richter scaling law in MIS go back to 1987 when, to the best of our knowledge, the first work showing this feature of MIS from coal mines in Poland was published (Kijko et al., 1987). The authors of this first work showed illustratively that the empirical distribution of magnitude maxima obeyed none of the three Gumbel's forms, which are the only possibilities for the asymptotic distribution of extremes (Gumbel, 1958) Instead, it was divided into two branches, which could be modelled by separate curves of the third Gumbel distribution type. The results led the authors to the conclusion that at least two seismic energy release modes, the high- and low-energy modes, were present in the studied seismic series. Other documented cases of the complexity of MIS magnitude distribution came from Indian gold mines (Subbaramu et al., 1989), a nickel mine in Canada (Trifu et al., 1993) and deep gold mines of South Africa (Feustel, 1997; Richardson and Jordan, 2002). Based on the observed distinct bimodality of the magnitude distribution, the authors of the last cited work suggested that this feature was due to a mixing of the two populations of events: fracture-dominated ruptures in a low normal-stress environment and frictiondominated ruptures. To study the observed multi-modality effects quantitatively, we applied the smoothed bootstrap test for multi-modality (Silverman, 1986; Efron and Tibshirani, 1998), modified for the exponential-like shape of magnitude distributions (Lasocki, 2001; Lasocki and Orlecka-Sikora, 2008). The test investigated two null hypotheses: H01 that the number of modes in the probability density of magnitude was no more than one and $\mathrm{H} 02$ that there was one or no bumps in the density. The mode is a local maximum of the density and the bump is an interval ((a) and (b)) such that the probability density is concave over (a) 
and (b) but not over any larger interval (Silverman, 1986). The low significance of either of these two hypotheses is evidence of the complexity of the underlying magnitude distribution and, in particular, its inconsistency with the Gutenberg-Richter relation. Altogether eight large magnitude samples from different mining panels of Polish copper mines were tested, for which in all eight cases the significance of H02 was less than 0.1, in six cases it was less than 0.05 , and in two cases the significance of H01 was less than 0.05 . Based on these results, we can conclude that the complexity feature of MIS magnitude distribution has been statistically acknowledged.

Usually the uncovered complexity features of the magnitude distribution are not strong enough to disqualify any use of the Gutenberg-Richter based model for magnitude. It is the author's opinion this model is a useful approximation and can be successfully applied for instance to monitor time variations of the seismic hazard in mines. Such applications have already proved their usefulness (Lasocki, 1993; Gibowicz and Lasocki, 2001). The Gutenberg-Richter model cannot, however, be used when a detailed modelling of the tail of magnitude distribution is required, which is the case of the stationary seismic hazard analysis. In such instances the slight differences between the model and actual distribution, caused by the distribution complexity, are magnified because the magnitude cumulative distribution is located in an exponent. The problem has been thoroughly studied in Lasocki (2001; 2005), Lasocki and Orlecka-Sikora (2008) and Orlecka-Sikora and Lasocki (2005) where the severity of errors resulting from an approach that neglects the complex features of magnitude distribution is discussed as well as the remedying approach.

\section{Discussion - consequences for predictability}

The process of the occurrence of seismic events in mines is non-stationary and the inter-event times are interrelated. The process of magnitude of these events is non-stationary and has both long and short memory. The same memory properties seem to hold for the inter-event distance; though studies of this parameterisation have not been completed yet (Węglarczyk, pers. comm., 2008). Certainly, these features complicate the statistical analysis of MIS data. On the other hand it is worth realising that the Poisson process of event occurrence and stationary, the memoryless processes of other event parameters mean that such events occur at random. Likewise, all event parameters are random selections from unchangeable distributions. The samples from such processes are statistical not stochastic and, being such, are convenient for statistical analyses. However, the maximum which can be achieved from such data are estimations of underlying distributions. Following, a prediction, understood as a forecast of future states of the processes is not possible because the states at all times oscillate in the same random way about constant expected values. Therefore the non-stationarity and inter-relations revealed by the presented analyses of mining seismic data, in addition to making the situation complicated, suggest that the mining seismic process is intrinsically predictable. The slow changes of this process permit one to treat it as quasi-stationary or segmental-stationary, which means that samples from limited time periods are underlain by approximately constant distributions. A seismic network with an appropriately low magnitude limit of recording can ensure that such samples will be big enough to determine the temporary states of the process.

The non-log-linear scaling of magnitude has two-fold consequences. On the one hand it means that the seismic energy of mining events does not obey the power law, which contradicts the thesis that the process is unpredictable because it is chaotic in the deterministic sense. On the other hand we are in fact interested in predicting larger events, whose magnitudes belong to the second, less numerous component of the multicomponental magnitude process. It is impossible to collect numerous enough samples from this component in time periods of a length that ensure the relative stationarity of the process. Memory in the magnitude process, which suggests the possibility that information on larger magnitudes can be drawn from smaller ones, and a model free estimation of the magnitude distribution (Kijko et al., 2001) can help to solve this problem. 


\section{Conclusions}

A detailed insight into the statistical properties of MIS shows that the MIS generation process is complex:

- The MIS is transient. MIS forms well-defined zones that correlate in space and time with mining works.

- The process of seismic event occurrence in mines is time-dependent and at best can be treated as quasi-stationary. Smaller events play an important role in providing information on the non-stationarity.

- Event size parameterisation processes in MIS are time-dependent. Magnitudes are inter-related.

- The event-occurrence process, the event-magnitude process and also probably the event-location process have both long and short memory, i.e. inter-event times, inter-event distances and magnitudes are internally inter-related.

- One of possible mechanisms of interaction of mining-induced seismic events is the static Coulomb stress transfer.

- The populations of magnitudes of mining seismic events consist of at least two components. The magnitude distribution is complex and often multi-modal. The distribution cannot be accurately approximated with the Gutenberg-Richter model.

The complexity of the MIS generation process complicates the practice of the statistical analysis of mining seismic data. On the other hand, however, non-stationarity and inter-relations mean that the mining seismic event generation process is intrinsically predictable.

\section{Acknowledgements}

This work was prepared within the framework of a research project No. PBS-Grecja/10/2007, financed by the Ministry of Education and Science of Poland during 2007 to 2009.

\section{References}

Chinnery, M.A. (1963) The state of stress changes that accompany strike-slip faulting, Bulletin of the Seismological Society of America, Vol. 53, 921-932.

Du Toit, C. and Mendecki, A.J. (2007) Examples of time distribution of seismic events in mines, Our Changing Planet. Proceedings IUGG XXIV General Assembly Perugia, Italy 2007, Published on website: www.iugg2007perugia.it, Abstract 6609.

Efron, B. and Tibshirani, R.J. (1998) An Introduction to the Bootstrap, Chapman and Hall, London.

Feustel, A.J. (1997) Temporal-spatial $b$-values and observed relationships to structurally controlled ground falls in an open-stope mine, Proceedings of the 4th International Symposium on Rockburst and Seismicity in Mines, Gibowicz, S.J. and Lasocki, S. (editors), Balkema, Netherlands, pp. 191-195.

Gardner, J.K., and Knopoff, L. (1974) Is the sequence of earthquakes in southern California, with aftershocks removed, Poissonian Bulletin of the Seismological Society of America, Vol. 64, pp. 1363-1367.

Gibowicz, S.J. and Lasocki, S. (2001) Seismicity induced by mining: Ten years later. Advances in Geophysics, Vol. 44, pp. 39-181.

Gomberg, J., Belardinelli, M.E., Cocco, M. and Reasenberg, P. (2005) Time-dependent earthquake probabilities, Journal of Geophysical Research. Vol. 110, B05S04, doi:10.1029/2004JB003405.

Gumbel, E.J. (1958) Statistics of Extremes, Columbia University Press, New York.

Hurst, H.E. (1951) Long-term storage capacity of reservoirs, Transactions of the American Society of Civil Engineers, $116 \mathrm{p}$.

Kagan, Y.Y. (1999) Universality of seismic moment-frequency relation, Pure and Applied Geophysics, Vol. 155, pp. 537-573.

Kijko, A. (1997) Keynote lecture: Seismic hazard assessment in mines, Proceedings of the 4th International Symposium on Rockburst and Seismicity in Mines, Gibowicz, S.J. and Lasocki, S. (editors), Balkema, Netherlands, pp. 247-256.

Kijko, A., Drzęźla, B. and Stankiewicz, T. (1987) Bimodal character of the distribution of extreme seismic events in Polish mines, Acta Geophysica Polonica, Vol. 35, pp. 157-166. 
Kijko, A., Lasocki, S. and Graham, G. (2001) Nonparametric seismic hazard analysis in mines,. Pure and Applied Geophysics, Vol. 158, pp. 1655-1676.

King, G.C.P. and Cocco, M. (2001) Fault interaction by elastic stress changes: new clues from earthquake sequences, Advances in Geophysics, Vol. 44, pp. 1-38.

Lasocki, S. (1992) Non-Poissonian structure of mining-induced seismicity, Acta Montana, Vol. 84, pp. 51-57.

Lasocki, S. (1993) Statistical prediction of strong mining tremors, Acta Geophysica Polonica, Vol 41, pp. $197-234$.

Lasocki, S. (2001) Quantitative evidences of complexity of magnitude distribution in mining-induced seismicity: implications for hazard evaluation. Rockburst and Seismicity in Mine: Dynamic Rock Mass Response to Mining, G. van Aswegen, R.J. Durrheim and W.D. Ortlepp (editors.), SAIMM S27, Johannesburg, pp. 543-550.

Lasocki, S. (2005) Probabilistic analysis of seismic hazard posed by mining induced events, Controlling Seismic Risk, Proceedings Sixth International Symposium on Rockburst and Seismicity in Mines 9-11 March, Australia, Y. Potvin and M. Hudyma (editors), Australian Centre for Geomechanics, Perth, pp. 151-156.

Lasocki, S. and Orlecka-Sikora, B. (2008) Seismic hazard assessment under complex source size distribution of mininginduced seismicity. Tectonophysics in Press, doi:10.1016/j.tecto.2006.08.013.

Lomnitz, C. (1974) Global Tectonics and Earthquake Risk, Elsevier Scientific Publishing Company, New York.

McGarr, A. and Simpson, D. (1997) A broad look at induced and triggered seismicity, Rockburst and Seismicity in Mines, S.J. Gibowicz and S. Lasocki (editors), Balkema, Rotterdam, pp. 385-396.

Orlecka-Sikora, B. and Lasocki, S. (2002) Clustered structure of seismicity from the Legnica-Glogow copper district, Publications of the Institute of Geophysics Polish Academy of Sciences, Vol. M-24 502 (340), pp. 105-119 (in Polish with English abstract).

Orlecka-Sikora, B. and Lasocki, S. (2005) Nonparametric characterization of mining induced seismic sources, Controlling Seismic Risk, Proceedings Sixth International Symposium on Rockburst and Seismicity in Mines 9-11 March, Australia, Y. Potvin and M. Hudyma (editors), Australian Centre for Geomechanics, Nedlands, pp. 555-560.

Orlecka-Sikora, B., Papadimitriou, E. and Kwiatek, G. (2008) Study of the interaction among mining induced seismic events in the Legnica-Glogow Copper District, Poland, Acta Geophysica (submitted).

Reasenberg, P.A. and Simpson, R.W. (1992) Response of regional seismicity to the static stress change produced by the Loma Prieta earthquake, Science, Vol. 255, pp. 1687-1690.

Richardson, E. and Jordan, T.H. (2002) Seismicity in deep gold mines of South Africa: Implications for tectonic earthquakes, Bulletin of the Seismological Society of America, Vol. 92, pp. 1766-1782.

Silverman, B.W. (1986) Density Estimation For Statistics And Data Analysis. Monographs on Statistics and Applied Probability, Chapman and Hall, London.

Smith, S.W. and Van de Lindt, W. (1969) Strain adjustments associated with earthquakes in Southern California, Bulletin of the Seismological Society of America, Vol. 59, pp. 1569-1589.

Steacy, S., Gomberg, J. and Cocco, M. (2005), Introduction to special section: Stress transfer, earthquake triggering, and time-dependent seismic hazard, Journal of Geophysical Research, Vol. 110, B05S01, doi:10.1029/2005JB003692.

Stein, R.S. and Lisowski, M. (1983) The 1979 Homestead Valley earthquake sequence, California: Control of aftershocks and postseismic deformation, Journal of Geophysical Research, Vol. 88, pp. 6477-6490.

Subbaramu, K.R., Rao, B.S.S., Krishnamurthy, R. and Srinivasan, C. (1989) Seismic investigations of rockbursts in the Kolar Gold Fields, Proceedings 4th Conference on Acoustic Emission/Microseismic Activity in Geologic Structures and Materials, Penn State University, H.R. Hardy, Jr. (editor), Clausthal, Trans Tech Publications, pp. 265-274.

Trifu, C-I., Urbancic, T.I. and Young, R.P. (1993) Non-similar frequency-magnitude distribution for $m<1$ seismicity, Geophysical Research Letters, Vol. 20, pp. 427-430.

Węglarczyk, S. and Lasocki, S. (2008) Studies of long and short memory in mining-induced seismic processes. Acta Geophysica (submitted). 